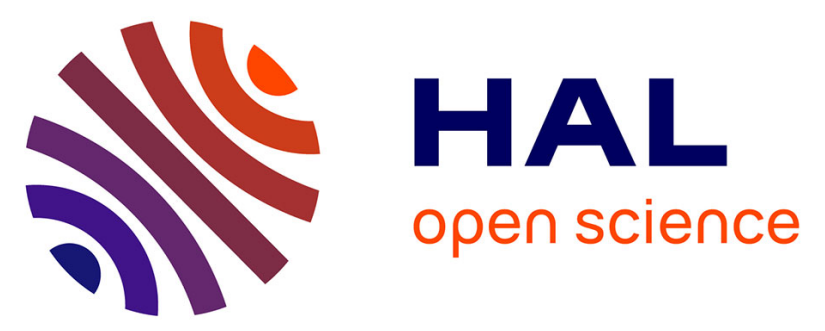

\title{
Resistance to Plasmopara viticola in grapevine 'Bianca' is controlled by a major dominant gene causing localised necrosis at the infection site
}

\author{
Diana Bellin, Elisa Peressotti, Didier Merdinoglu, Sabine \\ Merdinoglu-Wiedemann, Anne-Francoise Adam-Blondon, Guido Cipriani, \\ Michele Morgante, Raffaele Testolin, Gabriele Di Gaspero
}

\section{To cite this version:}

Diana Bellin, Elisa Peressotti, Didier Merdinoglu, Sabine Merdinoglu-Wiedemann, Anne-Francoise Adam-Blondon, et al.. Resistance to Plasmopara viticola in grapevine 'Bianca' is controlled by a major dominant gene causing localised necrosis at the infection site. TAG Theoretical and Applied Genetics, 2009, 120 (1), pp.163-176. 10.1007/s00122-009-1167-2 . hal-01137023

\author{
HAL Id: hal-01137023 \\ https://hal.science/hal-01137023
}

Submitted on 13 Jun 2015

HAL is a multi-disciplinary open access archive for the deposit and dissemination of scientific research documents, whether they are published or not. The documents may come from teaching and research institutions in France or abroad, or from public or private research centers.
L'archive ouverte pluridisciplinaire $\mathbf{H A L}$, est destinée au dépôt et à la diffusion de documents scientifiques de niveau recherche, publiés ou non, émanant des établissements d'enseignement et de recherche français ou étrangers, des laboratoires publics ou privés. 


\title{
Resistance to Plasmopara viticola in grapevine 'Bianca' is controlled by a major dominant gene causing localised necrosis at the infection site
}

\author{
Diana Bellin • Elisa Peressotti - Didier Merdinoglu • Sabine Wiedemann-Merdinoglu • \\ Anne-Françoise Adam-Blondon • Guido Cipriani • Michele Morgante • \\ Raffaele Testolin • Gabriele Di Gaspero
}

Received: 7 July 2009/ Accepted: 27 September 2009

(C) Springer-Verlag 2009

\begin{abstract}
Downy mildew resistance is a quantitative trait in grapevines of the genus Vitis. The grapevine 'Bianca' has retained resistance, originally present in its North American ancestors, through several cycles of backcrossing with susceptible cultivars of Vitis vinifera followed by phenotypic selection. The genetic control of the trait was studied using 116 full-siblings from the cross 'Chardonnay' $\times$ 'Bianca' and parental genetic maps consisting of 298 and 312
\end{abstract}

Communicated by M. Xu.

Electronic supplementary material The online version of this article (doi:10.1007/s00122-009-1167-2) contains supplementary material, which is available to authorized users.

D. Bellin · E. Peressotti · G. Cipriani · M. Morgante ·

R. Testolin · G. Di Gaspero $(\square)$

Dipartimento di Scienze Agrarie e Ambientali,

University of Udine, via delle Scienze 208, 33100 Udine, Italy

e-mail: gabriele.digaspero@uniud.it

E. Peressotti · D. Merdinoglu $\cdot$ S. Wiedemann-Merdinoglu Institut National de la Recherche Agronomique,

UMR1131 Santé de la Vigne et Qualité du Vin,

28 Rue de Herrlisheim, 68021 Colmar, France

D. Merdinoglu $\cdot$ S. Wiedemann-Merdinoglu

Université de Strasbourg, UMR1131, 67000 Strasbourg, France

A.-F. Adam-Blondon

UMR de Génomique Végétale, INRA-CNRS-UEVE,

2, Rue Gaston Crémieux, CP5708, 91057 Evry Cedex, France

M. Morgante $\cdot$ R. Testolin · G. Di Gaspero

Istituto di Genomica Applicata, Parco Scientifico e Tecnologico

Luigi Danieli, via Jacopo Linussio 51, 33100 Udine, Italy

Present Address:

D. Bellin

Dipartimento di Biotecnologie, University of Verona,

Strada Le Grazie, 15, 37134 Verona, Italy markers, respectively. Ratings of resistance and histological identification of the stage of interaction, when pathogen development is impaired in resistant individuals, were performed using leaf disc inoculation assays with two isolates of Plasmopara viticola collected in Italian and French vineyards. 'Bianca' and 59\% of its offspring were heterozygous for a dominant gene, located in a $2.9 \mathrm{cM}$ interval at the Rpv3 locus on chromosome 18, responsible for the onset of a hypersensitive response (HR) at the infection sites within 2 days post inoculation (dpi). Localised necrosis was the earliest phenotypic difference compared to susceptible individuals, it did not halt pathogen growth, but it was associated with a significant reduction of pathogen performance and disease symptoms from 3 to 6 dpi. QTL peaks for quantitative ratings revealed the strongest effects being caused by the Rpv3 locus: extent of mesophyll colonisation (LOD 3.1, percentage of explained phenotypic variance $16.2 \%)$, sporulation density $(29.7,74.3 \%)$, and symptom severity expressed by the OIV452 descriptor recommended by the Office International de la Vigne et du Vin (28.3, $74.6 \%$ ). Strong correlation was observed between the ability of a seedling to mount an HR under controlled experimental conditions and quantitative resistance of the adult plant exposed to natural infections in the field, which was expressed by the number of leaves with fungal sporulation, in two consecutive years of observations.

Keywords Downy mildew · NBS-LRR .

Quantitative resistance - QTL analysis · Vitis vinifera

\section{Introduction}

Plasmopara viticola (Berk. \& M. A. Curtis) Berl. \& De Toni is a biotrophic pathogen which causes downy mildew 
to members of the family Vitaceae, in particular to the cultivated species Vitis vinifera. $P$. viticola is native to the Southeastern United States, and was inadvertently introduced into Europe in the second half of the nineteenth century. $V$. vinifera and $V$. acerifolia are the only grape species in which all of the evaluated accessions were found invariably susceptible (Cadle-Davidson 2008), whereas resistant individuals are intermixed with susceptible individuals in many species that grow in wild environments in North America. North American grapes exhibit host resistance against $P$. viticola, which is mounted after the haustorium has established contact with the membrane of mesophyll cells (Díez-Navajas et al. 2008; Jürges et al. 2009). Defence reactions occur with variable promptness and magnitude during the latency stage, the period following colonisation of the leaf, during which hyphae grow in the inner side of the lamina without causing visible signs of the disease. Timing and intensity of plant reaction have a large genotypic component. Most ratings of resistant accessions were consistent among trials conducted under different experimental conditions (Staudt and Kassemeyer 1995; Cadle-Davidson 2008). However, phenotypic values might be influenced by environmental effects, such as concentration of the inoculum, virulence of the isolate, relative humidity and temperature, and any other factor that affects the physiological state of the experimental plants prior to and during the assay. Resistant accessions within the North American species V. riparia, V. cinerea, $V$. labrusca, V. rupestris, V. berlandieri, V. lincecumii, and Muscadinia rotundifolia exhibit variable levels of resistance (Dai et al. 1995; Kortekamp and Zyprian 2003; Unger et al. 2007; Díez-Navajas et al. 2008). All resistant accessions in North American Vitis species allow P. viticola to complete its life cycle, though sporangia are released at a lower rate than in susceptible individuals. Some genotypes of the related genus Muscadinia more efficiently halt hyphal growth during mesophyll colonisation and do not manifest visible symptoms outside the leaf, particularly sporulation; while some exotic Asian grapes support hyphal growth only on the outer side of the leaf lamina, preventing stomata penetration and the release of viable sporangiophores (Jürges et al. 2009).

Resistant grapes from North America have been extensively crossed with $V$. vinifera in order to introgress resistance into fruiting cultivars. 'Bianca' is a $V$. vinifera backcross of 'Villard blanc', selected in the 1960s (Csizmazia and Bereznai 1968). 'Villard blanc', also known as Seyve Villard 12-375, transmits to the progeny the highest level of downy mildew resistance (Iwanov and Waltschev 1973; Cadle-Davidson 2008) among many hundred hybrids of the series 'Seibel' and 'Seyve Villard', produced by the French breeding efforts for the introduction of North American resistance genes into the $V$. vinifera background.
The resistant ancestors of 'Villard blanc' include accessions of five Vitis species, namely V. aestivalis, V. berlandieri, V. cinerea, lincecumii, and V. rupestris. 'Bianca' shares ancestors with other well-studied resistant grapevine varieties, such as 'Regent'; a full sibling of 'Villard Blanc' is one of the grandparents of 'Regent'. 'Regent' might also have inherited additional resistance factors from the lineage of the other resistant grandparent 'Chancellor', synonym of 'Seibel 7053' (Eibach and Töpfer 2003).

Three loci with major effects on downy mildew resistance are emerging from comparative genetic mapping across grapevine genomes, namely Rpv1, Rpv2, and Rpv3 (Merdinoglu et al. 2003; Fischer et al. 2004; Welter et al. 2007; Wiedemann-Merdinoglu et al. submitted and the present paper). Rpvl is located on M. rotundifolia chromosome (chr) 12 and the responsible gene, still unidentified in a cluster of NBS-LRR candidates, is genetically associated to Run1, a locus conferring powdery mildew resistance (Barker et al. 2005; Wiedemann-Merdinoglu et al. submitted). A QTL peak in the same region was identified in $V$. riparia, which explained the reduction of sporangia released per unit of leaf area (Marguerit et al. 2009). The Rpv2 locus is located on chr18, and its precise interval has been identified in $M$. rotundifolia by Wiedemann-Merdinoglu et al. (submitted). A further locus downstream of $R p v 2$, in the distal part of $\operatorname{chr} 18$, is described in the present paper and has been given the name $R p v 3$. The Rpv3 locus was first identified by interval mapping in 'Regent', where it explained up to $56 \%$ of the phenotypic variance in 4 years of field observations (Fischer et al. 2004; Welter et al. 2007). The unstable position of the QTL peak across different years of observation and the wide confidence interval led the authors to assume that more than one functional gene might reside at the Rpv3 locus. Either the same or other functional resistance genes/alleles at the Rpv3 locus might be present in 'Villard Blanc' (Zyprian et al. 2005). Based on the interval of confidence in the 'Regent' and 'Villard Blanc' maps, it was unclear whether the Rpv3 QTL is due to a different resistance gene that can be genetically mapped apart from the M. rotundifolia Rpv2 locus, at the resolution provided by the available mapping populations, or if it is an allelic QTL, or the underlying genes, each other functional in M. rotundifolia or Vitis, are linked within a short physical distance.

Weak QTLs associated with minor effects have also been identified in specific experiments and genotypes, which have provided an indication of many genetic factors segregating from heterozygous grapes, each one potentially affecting steps along resistance pathways or influencing the physiological steady-state of the host. QTLs fluctuating above and below the threshold of significance were identified on chr7 in V. riparia (Marguerit et al. 2009) and in 
'Villard Blanc' (Zyprian et al. 2005). Minor QTLs were also found in genotypes of $V$. riparia on chr9 (Marguerit et al. 2009), and in 'Regent' on chr4 and chr5 (Welter et al. 2007).

In this paper, we report phenotypic and genetic dissection of downy mildew resistance inherited by 'Bianca' from 'Villard Blanc' and transmitted to its resistant offspring, when crossed with the susceptible grapevine 'Chardonnay'. QTL analysis and mapping of quantitative and qualitative components of plant reaction and pathogen performance are based on several phenotypic ratings that are scored using leaf disc assays on plants of the progeny grown in a controlled environment (Díez-Navajas et al. 2008). The reliability of genetic determinants identified under those experimental conditions was confirmed by QTL analysis in the same progeny, using quantitative scores on intact leaves artificially inoculated outdoors in potted plants, and on whole plants exposed to natural infections in a standard field production.

\section{Materials and methods}

Plant material and experimental design

The segregation of resistance to downy mildew was studied in 116 offspring of the cross 'Chardonnay' $\times$ 'Bianca'. Vitis vinifera 'Chardonnay' is susceptible to downy mildew and Vitis 'Bianca' is a resistant grapevine, obtained by hybridization of $V$. vinifera 'Bouvier' and 'Villard Blanc' (Csizmazia and Bereznai, 1968). Original seedlings of the 'Chardonnay' $x$ 'Bianca' populations were grown in the field, and six biological replicates per seedling were propagated from budwood cuttings. For field experiments, budwood was grafted onto Kober 5BB and two replicated plants per individual were planted in vineyards in 2005, trained to the Guyot system at a density of 4,000 vine$\mathrm{s} \mathrm{ha}^{-1}$, with $1 \mathrm{~m}$ within-row spacing. Scores of naturally infected plants were taken in the seasons of 2007 and 2008. For artificial inoculations, the same seedlings were propagated from cuttings and grown on their adventitious roots in potted soil. Two replicates per individual were grown outdoors in the seasons of 2004 and 2005 and artificially inoculated. These plants were thus exposed to natural conditions prior to inoculation and during incubation, and the inoculated leaf remained attached to the shoot for the entire course of the experiment. Finally, two replicates per individual were grown in a greenhouse for controlled artificial inoculations on leaf discs from detached leaves. A graphical overview of the experimental design, the type of plant material, the experimental conditions before and during infection, and the parameters used for expressing the level of disease resistance are given in Supplementary Material S1.
Plasmopara viticola isolates for artificial inoculation

Infected leaves at the oil spot stage were collected from $V$. vinifera vineyards in North-Eastern Italy (Udine, $46^{\circ} 02^{\prime} \mathrm{N}$, $13^{\circ} 13^{\prime} \mathrm{E} ; 88 \mathrm{~m}$ asl) and Eastern France (Colmar, $48^{\circ} 03^{\prime}$ $\mathrm{N}, 7^{\circ} 20^{\prime} \mathrm{E} ; 406 \mathrm{~m}$ asl). These two sources of inoculum are referred to below as NE-I isolate and E-F isolate. Sporangia were obtained after incubation of detached leaves at $20^{\circ} \mathrm{C}$, $100 \% \mathrm{RH}$ in the dark. The NE-I isolate was propagated as a mixed culture of $P$. viticola sporangia. The E-F isolate was propagated as a monosporangial culture (Díez-Navajas et al. 2008). Isolates were maintained and propagated by spraying a 50,000 sporangia $\mathrm{ml}^{-1}$ suspension on susceptible plants of $V$. vinifera, grown and incubated while isolated in a growth chamber at $21^{\circ} \mathrm{C}$, with day length of $16 \mathrm{~h}$, until sporulation. Fresh sporangia were dried, stored at $-20^{\circ} \mathrm{C}$ and re-hydrated with distilled water at $4^{\circ} \mathrm{C}$ for $2 \mathrm{~h}$ prior to inoculation.

Phenotypic analysis on artificially inoculated leaf discs

\section{Artificial inoculation of leaf discs and incubation under controlled conditions}

For each individual, the fourth and fifth leaves beneath the shoot apex were detached from two replicated plants grown in the greenhouse and rinsed with water. Sixteen leaf discs of 1-cm diameter were excised from the four leaves with a cork borer and plated onto wet paper in Petri dishes with the abaxial side up. Discs were sprayed with $P$. viticola inoculum suspension at 150,000 sporangia $\mathrm{ml}^{-1}$. Petri dishes were incubated in a growth chamber at $21^{\circ} \mathrm{C}$ and day length of $16 \mathrm{~h}$, for 6 days. This procedure and all subsequent ratings (see below) were repeated twice using the E-F isolate. Inoculation with the NE-I isolate was conducted in the same way, except that the leaves were sampled from field grown plants and leaf discs were soaked in 50,000 sporangia ml $\mathrm{ml}^{-1}$ suspension for $2 \mathrm{~h}$ (Supplementary Material S1).

\section{Ratings of plant reaction and pathogen performance in leaf} disc assays

Disease progression was monitored daily and quantified based on observations of the plant reaction and on measurement of parameters related to pathogen performance. Ratings based on steady-state scores and observations at each time point were used for genetic analysis. For each parameter, the scores were also integrated over the course of disease progression and used as cumulative data (referred to as progression curve, PC).

Plant reaction was scored as the presence or absence of visible necrosis from 2 to 6 days post infection (dpi). Leaf 
discs of individuals in which necrotic spots were not detectable to the naked eye were also observed under a light microscope to discriminate between the occurrence of substomatal necrosis limited to a few cells, and complete absence of necrotic reaction.

The extent of sporulation was rated in four ways, two of which by assigning categorical values upon visual inspection, the third by quantitatively counting the sporangia produced per unit of leaf area, and the fourth by measuring sporangia size. First, the leaf disc area yielding sporangiophores was assessed by visual inspection from 3 to $6 \mathrm{dpi}$, rated by assigning a value from 1 to 9 based on the extent of sporulating area across leaf discs ( 1 most extended, 9 most restricted; see Table 1 for details on each class) and expressed by the OIV452 descriptor adapted to the leaf disc assay. A cumulative rating was obtained by summing the scores from 3 to 6 dpi (OIV452_PC). Second, sporulation density was scored at $5 \mathrm{dpi}$ (SD_5dpi) by assigning a value from 1 to 9 (1 high sporulation density; 9 few, sparse, and restricted sporulation), based on the density of sporangiophores within the leaf disc sectors yielding sporulation, irrespectively of the total area covered by sporulation. Third, sporangia were counted at 6 dpi and expressed as a concentration per unit of leaf area. Sporangia were collected from 10 leaf discs per individual by vortexing discs in $10 \mathrm{ml}$ of distilled water for $1 \mathrm{~min}$. One millilitre of suspension was used to determine sporangia concentration and sporangia size using a Z2 Coulter cell counter (Beckman Coulter) in the experiment with the E-F isolate. In the replicates inoculated with the NE-I isolate, $10 \mu \mathrm{l}$ of sporangia suspension was loaded onto a Bürker counting chamber and sporangia were counted under light microscope.

Magnitude of plant reaction and level of sporulation per individual were concomitantly rated by a visual index, the OIV452 descriptor recommended by the Office International de la Vigne et du Vin (Anonymous 1983) and adapted to the disc assay. Categorical values from 1 (the most susceptible) to 9 (the most resistant) were assigned based on the absence or presence of necrotic reactions and their size, as well as on the extent of sporulating area (see Table 1 for details on each class).

The extent of mesophyll invasion by the developing hyphae was assessed at 4 dpi using two leaf discs per individual (later referred to as MYCELIUM GROWTH 4dpi). Leaf discs were soaked in 5\% KOH until chlorophyll was removed. Mycelium inside the host mesophyll was stained with $0.05 \%$ aniline blue and discs were observed under a fluorescence microscope (Díez-Navajas et al. 2007). Categorical values ranging from 1 (mycelium present in all discs, intercostal fields of the mesophyll completely filled with mycelium) to 9 (primary hyphae not developing into a mycelium) were assigned upon visual inspection.

Phenotypic analysis on artificially inoculated vines grown outdoors in potted soil

Two replicates per individual were grown in potted soil in the spring seasons of 2004 and 2005, outdoors under natural conditions except for supplemental watering using drip emitters and for a $12 \%$ reduction of solar radiation due to overhead hail-protecting net. Vines were trellised in a single vertical shoot system. At the stage of 10-leaf shoot, the fourth fully expanded leaf was inoculated with the NE-I isolate of $P$. viticola, by spraying sporangia suspension at sunset, and wrapping the leaf into a sealed plastic bag overnight. Incubation occurred under natural conditions at the site of the experiment $\left(46^{\circ} 02^{\prime} \mathrm{N}, 13^{\circ} 13^{\prime} \mathrm{E}\right.$; $88 \mathrm{~m}$ asl), except for humidity in the canopy that was maintained at a high level by automatic watering of the foliage with overhead sprinklers during daylight hours. Individuals were scored for the presence or absence of sporulation by visual inspection. The scoring was repeated weekly during a 3-week period after inoculation. Artificial inoculation was repeated twice a year, in June and in July on newly developed leaves, for two consecutive years. The sum of sporulation records per genotype per replicate per inoculation was used for QTL analysis ( $\Sigma$ SPORULATION_2004, $\Sigma$ SPORULATION_2005).

Phenotypic analysis on naturally infected vines in the vineyard

Two replicates per individual were grown in open field. The experimental vineyard was conducted following standard production practices, except for withholding the application of fungicides specific for or with residual
Table 1 Ratings of downy mildew resistance based on the OIV452 index (Anonymous 1983), adapted to scoring phenotypes in the leaf disc assay used in this study

Interclass scoring, such as 2, 4, etc., was allowed

\begin{tabular}{lll}
\hline Class & Sporulation & Plant reaction \\
\hline 1 & Sporangiophores densely cover the whole disc area & Absence of necrosis \\
3 & Predominant patches of dense sporulation & Absence of necrosis \\
5 & Patches of sparse sporulation equally intermixed & Necrotic flecks underneath sporulating areas \\
& with asymptomatic areas & \\
7 & Small spots with sparse sporangiophores & Necrotic spots or substomatal necrosis \\
9 & Absence of sporangiophores & Necrotic spots or substomatal necrosis
\end{tabular}


activity against $P$. viticola until the occurrence of natural primary infections. Daily minimum, maximum, and average temperatures, $\mathrm{RH}$, and rainfall were recorded at the experimental site during the period of observation and are given in Supplementary Material S2. Disease severity was quantitatively expressed by the number of leaves showing sporulation. To identify the appropriate time for rating, the progeny was scored every day after the appearance of the first oil spot. The final rating of the number of leaves with sporulating lesions per individual was used for QTL analysis. Data collected on June 13, 2007 and May 30, 2008 (No.SPO.LEAVES_2007, No.SPO.LEAVES_2008) were averaged over two biological replicates and included in QTL analysis. The vineyard was then sprayed with fungicides to prevent defoliation of susceptible plants.

\section{Statistical analysis}

Two biological replicates were independently scored in all experiments. The reproducibility of each rating and the genotypic effect were evaluated by a one-way ANOVA test. Values averaged over biological replicates and experiments were used for QTL analysis.

\section{Mapping of qualitative traits and QTL analysis}

Genotypic data of the 116 offspring of the cross 'Chardonnay' $x$ 'Bianca' used for phenotyping were extracted from the dataset of a larger mapping population of 358 individuals (Cipriani et al. 2009). Parental and consensus maps were constructed using JoinMap 4.0. Each marker was tested for goodness of fit to the expected Mendelian segregation using the $\chi^{2}$ test. Distorted markers were maintained unless they affected marker order. Linkage groups were built at a LOD threshold of 4.0, and numbered according to Doligez et al. (2006). The best marker order was calculated using a regression mapping algorithm, and map distances were calculated in Kosambi cM. Marker orders were obtained with the first or the second round of ordering performed by JoinMap, and those with the lowest chi-square values were selected (Supplementary Material S3). The HR trait, that is presence/absence of visible necrotic spots or localised necrosis at substomatal cells, was included in the genotypic dataset and mapped as a Mendelian trait.

QTL analysis

QTL analysis was performed using interval mapping and multiple-QTL mapping algorithms of MapQTL 5.0 (Van Ooijen 2004). A non-parametric Kruskal-Wallis test was also performed. For all quantitative ratings, values averaged over biological replicates within each experiment and averaged over the experiments for replicated experiments were used for QTL analysis. LOD thresholds for significant QTLs at $\alpha=0.2$ and $\alpha=0.05$ were estimated using a permutation test with 1,000 permutations. The QTL peak was considered at a position associated with the maximum LOD score. The confidence interval of the map location for each QTL was defined as a one LOD support interval.

\section{Results}

Responses to $P$. viticola infection in leaf disc assays

The earliest reaction to $P$. viticola occurred in 'Bianca' within $48 \mathrm{~h}$ post infection, in the form of localised necrosis at the infection sites (Fig. 1). 'Chardonnay' did not exhibit any visible response to infection. After their appearance, necrotic spots at substomatal cells either enlarged to the surrounding cells, becoming visible to the naked eye, or in the case of a few individuals, remained only detectable with the aid of a microscope. Necrotic spots of either type appeared in $59 \%$ of the progeny at the same stage as in 'Bianca', while the remainder individuals were phenotypically similar to the susceptible parent. The ability of mounting an HR segregated as a Mendelian trait $\left(\chi^{2}=3.7\right.$ for 1 d.f.), with 'Bianca' heterozygous for a dominant allele controlling the HR and 'Chardonnay' homozygous for a recessive or non-functional allele. The observation of HR and the classification of the progeny based on this qualitative trait were facilitated by the artificial inoculation assay conducted on detached leaf discs from plants grown under controlled conditions in greenhouses. Conversely, a secure classification of the progeny based on the onset of HR was hampered in all plant material grown outdoors, due to the occurrence of aspecific necroses probably caused by other biotic and abiotic stresses across the population.

All individuals that developed necroses at the infection sites also displayed a significant reduction of mycelium growth in the mesophyll, assessed by staining and viewing the fungal structures at $4 \mathrm{dpi}$, compared to the susceptible parent and the other half of the progeny. As a consequence of this limitation in hyphal development, $P$. viticola completed its life cycle with the emergence of only sparse sporangiophores in the resistant individuals. By contrast, the susceptible individuals showed intercostal fields of the mesophyll completely colonised by mycelium at $4 \mathrm{dpi}$, and the same individuals supported $P$. viticola sporulation at a significantly higher extent than individuals that had mounted the HR.

Apart from the HR, all other parameters used as categorical or numerical scores of the phenotypes showed a continuous variation in the progeny. A significant 
Fig. 1 Plant reaction and Plasmopara viticola development in a 'Bianca' versus 'Chardonnay', and b two resistant offspring versus $\mathbf{c}$ two susceptible offspring. Leaf discs were laid with the abaxial side up. In the right column, fungal structures within the mesophyll were stained with aniline blue and shown as a cyan mass of hyphae using a fluorescence microscope. Resistant individuals developed localised necroses at the infection sites within the droplets of $P$. viticola suspension by 2 dpi. Sparse sporangiophores emerged at 4 and 6 dpi, mostly confined around the sites of attempted infection. Hyphae grew poorly within the intercostal field where the zoosporangia entered the mesophyll through stomata. Susceptible individuals did not show visible reactions and allowed the pathogen to sporulate as early as $4 \mathrm{dpi}$, after the mycelium had completely invaded the intercostal fields below the entry site of the germ tubes. In both compatible and incompatible interactions, hyphal growth was delimited by major veins

\section{A}
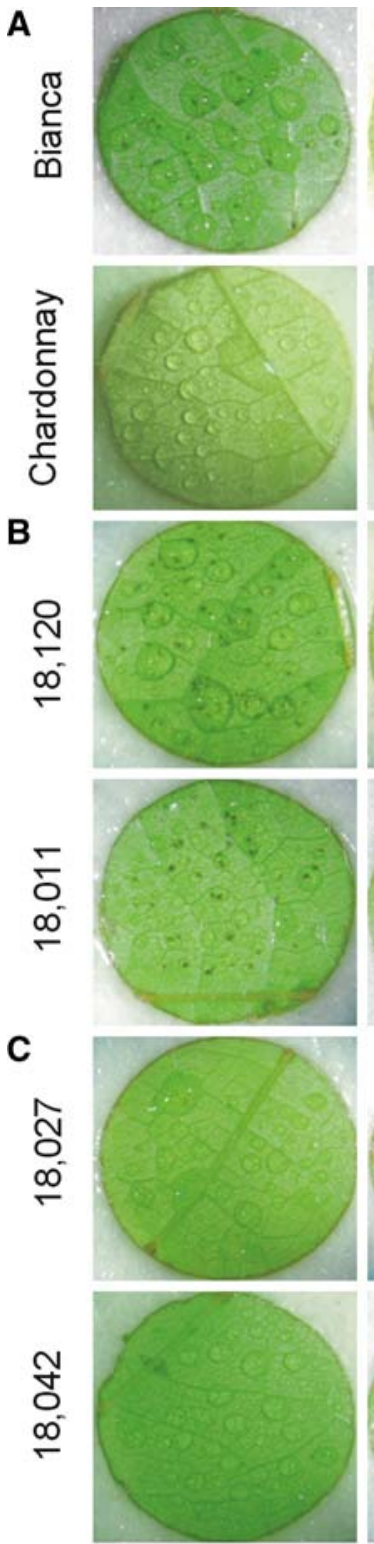

2 dpi
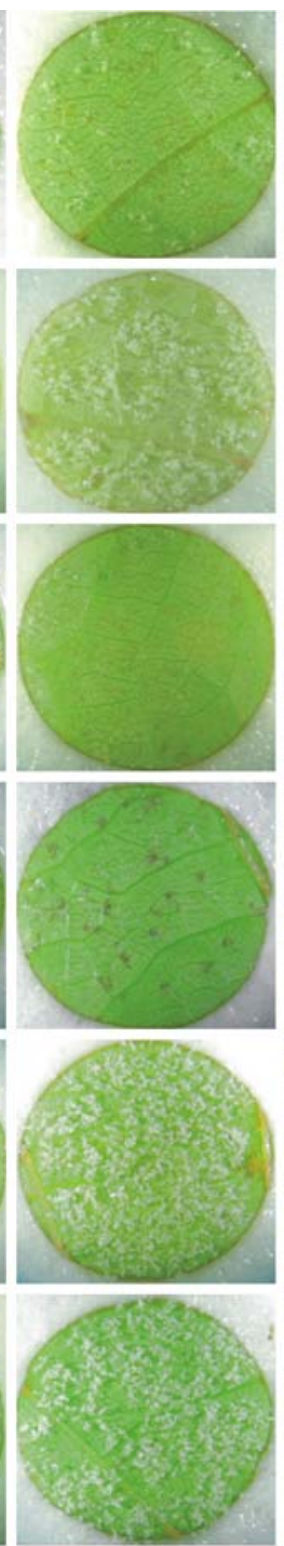

4 dpi
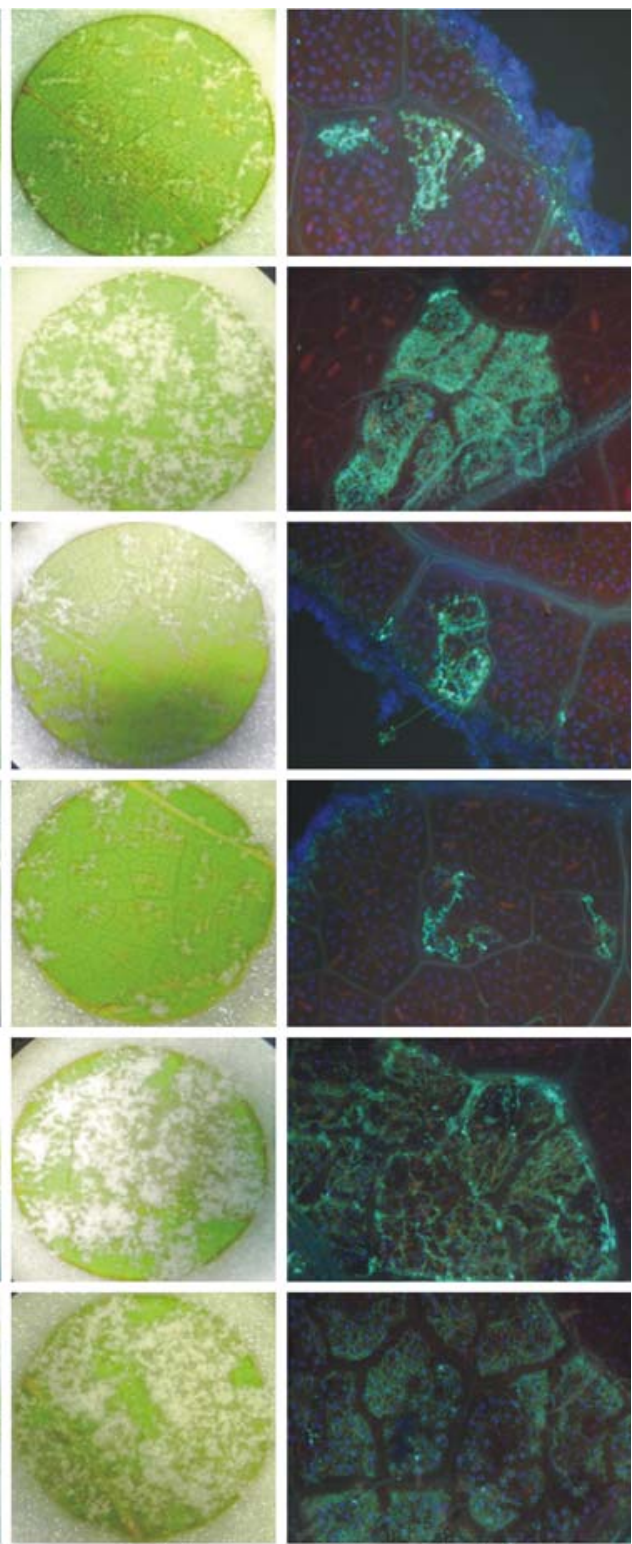

6 dpi

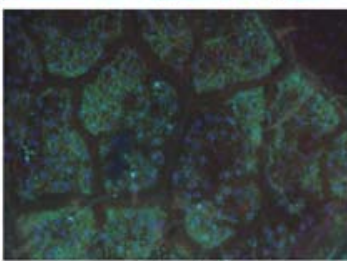

4 dpi genotypic effect on reduction of pathogen performance was detected every day from 3 to 6 dpi for the OIV 452 index, at 5 dpi based on categorical ratings of sporulation density, and for the number of sporangia released per unit of leaf area at $6 \mathrm{dpi}$, for inoculations of leaf discs from greenhouse-grown plants with the E-F isolate. In a similar way, pathogen development was significantly affected by the host genotype in inoculation of leaf discs from field grown plants challenged with the NE-I isolate for the extent of mesophyll colonisation by the mycelium at $4 \mathrm{dpi}$, and for the OIV452 index and for the number of sporangia released per unit of leaf area at $6 \mathrm{dpi}$. The size of the released sporangia was the only parameter in the leaf disc assay that was not affected by the genotype of the offspring.
Distribution of the F1 progeny based on quantitative ratings of pathogen growth

In the artificial inoculation assay of leaf discs from greenhouse-grown plants with the E-F isolate, the progeny was classified every day until 6 dpi using the OIV452 descriptor, for obtaining a dynamic description of the progress of infection in the population. The pathogen was at the latency stage in all individuals until 2 dpi. The first emergence of a few sporangiophores in some individuals was recorded at 3 dpi. At 3 dpi, the progeny showed a normal distribution for the extent of sporulation (OIV452 index, Fig. 2a). Most individuals displayed sparse sporangiophores confined in small leaf sectors and were rated 7. Class 7 included individuals that had already mounted a visible HR and 
Fig. 2 Distribution of F1 offspring of

'Chardonnay' $x$ 'Bianca' based on quantitative ratings of pathogen development in a the leaf disc assay, b the artificially inoculated intact leaves of potted plants, grown before inoculation and during latency outdoors under natural conditions, and $\mathbf{c}$ plants grown in an open field and exposed to naturally occurring infection. In $\mathbf{a}$, values are averaged over two biological replicates per individual and over two replicates of the whole experiment. The OIV452 index reflects the severity of downy mildew symptoms at each dpi (1 most susceptible, 9 most resistant), PCOIV452 reports the integration of the OIV452 index from 3 to $6 \mathrm{dpi}$. MYCELIUM GROWTH reports the extent of mesophyll invasion by the mycelium at 4 dpi, SPORANGIA $\mathrm{CM}^{-2}$ is the number of sporangia released at 6 dpi per unit of inoculated leaf area. In $\mathbf{b}$, values report the sum of scores recorded weekly during a 3week period after inoculation, averaged over two biological replicates, following two inoculations applied during the course of growing season. In $\mathbf{c}$, values are averaged over two biological replicates. Values of the parents 'Chardonnay' (Cha) and 'Bianca' (Bia) are indicated on top of the corresponding histogram
A
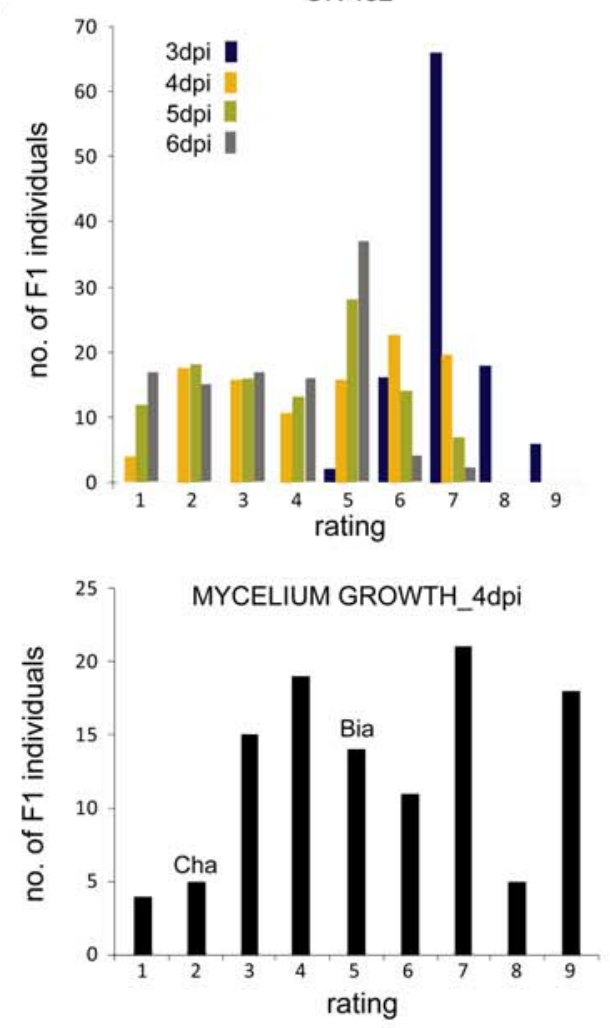

B ESPORULATION_outdoors2004
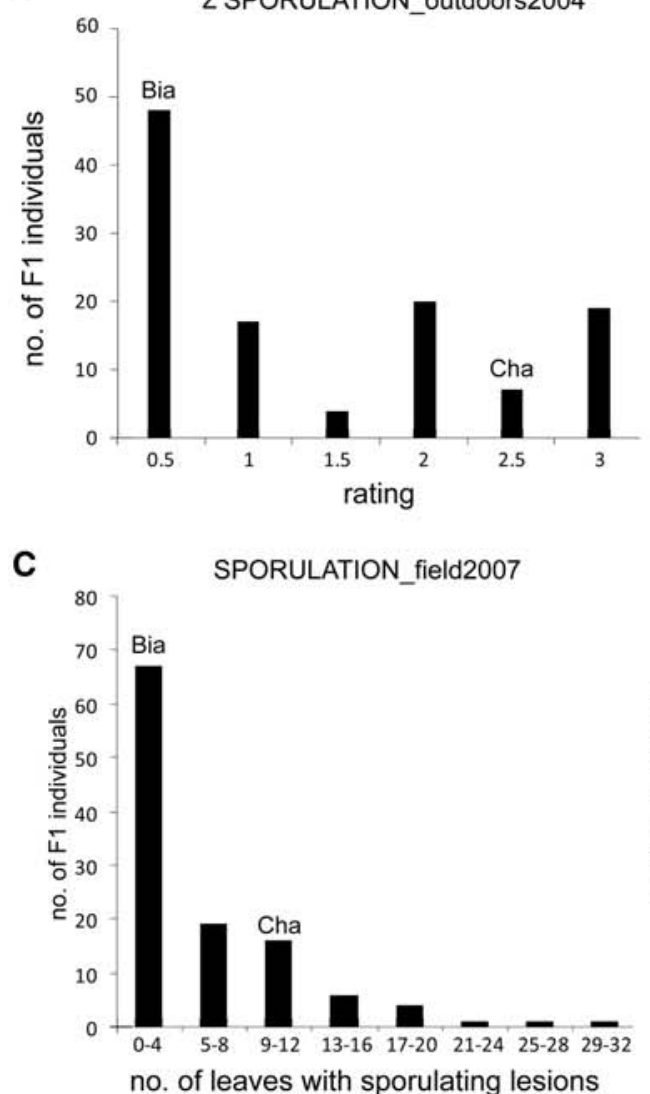

OIV452_PC
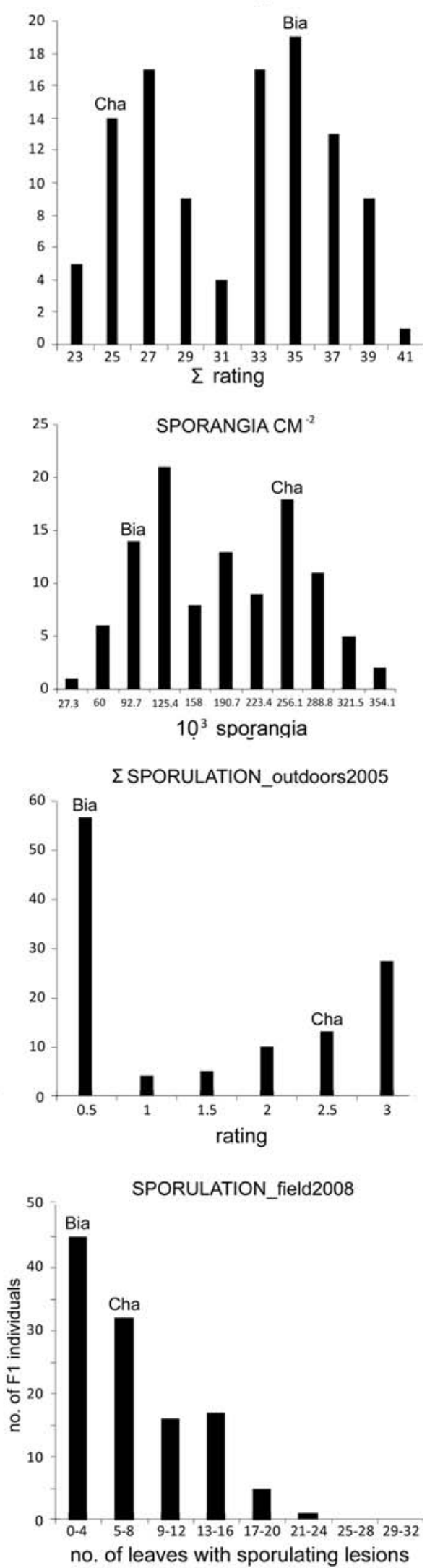
individuals that did not display any reaction. All individuals that fell into the OIV 452 classes 8 and 9 showed an HR, those individuals rated in classes 5 and 6 sporulated more extensively without any apparent host reaction. At 4 and $5 \mathrm{dpi}$, the shape of progeny distribution approximated a bimodal distribution, with half of the progeny peaking at classes 2 and 3 (predominant patches of dense sporulation, absence of necrotic reaction), and half peaking at classes 5 and 6 (spots of sparse sporulation, necrosis underneath sporulating segments). At 6 dpi, phenotypic values shifted to lower classes (more abundant sporulation) in all individuals, as a consequence of overwhelming advantages for pathogen development over defence barriers in the leaf disc assay. The bimodal distribution of downy mildew resistance based on the OIV452 descriptor was more evident when ratings for each individual were integrated over the course of disease progression from 3 to 6 dpi (Fig. 2a).

Sporulation density, a categorical score assigned upon visual inspection of sporangiophore density within the sporulating patches, was recorded at $5 \mathrm{dpi}$, the stage when the individuals could be attributed to each class with the best confidence (data not shown). Pathogen performance in terms of quantitative ability to propagate through sporulation on the host genotype, was numerically expressed by counting the sporangia released per unit of leaf surface at $6 \mathrm{dpi}$, in leaf discs from greenhouse and field grown plants (SPORANGIA $\mathrm{CM}^{-2}$ ). The progeny showed a bimodal distribution for all of these parameters. The correlation between visual inspection of sporulation density in leaf discs at $5 \mathrm{dpi}$, and counts of the number of sporangia per unit of leaf area at the end of the experiment showed a coefficient of correlation of -0.86 .

Finally, the categorical classification of the progeny based on the extent of mycelium growth at 4 dpi in the mesophyll of leaf discs from field grown plants resulted in $51 \%$ individuals assigned to classes 1-5 (intercostal fields of the mesophyll entirely or predominantly invaded by mycelium) and the remaining $49 \%$ rated in classes 6-9 (Fig. 2a). In the leaf disc assay from leaves detached from field grown plants, the coefficient of correlation between the extent of mycelium growth in the mesophyll at $4 \mathrm{dpi}$ and the OIV452 parameter at $6 \mathrm{dpi}$, which is mainly based on visible symptoms of the outer reproductive structure of the pathogen, was 0.72 .

To summarise, all quantitative scores in the leaf disc assays showed typical distributions observed for traits controlled by a single QTL with a strong dominant effect.

The scores of the progeny based on leaf disc assays performed on leaves from greenhouse-grown plants and inoculated with the E-F isolate were consistent with those obtained on leaves from field plants inoculated with the NE-I isolate. For each individual, scores recorded in leaf discs from field plants shifted consistently towards lower levels of pathogen development compared to leaf discs from greenhouse-grown plants, which could be caused by an inherent hardiness of leaf tissues grown outdoors, on plants exposed to harsher conditions. For instance, the average value of the progeny for pathogen sporulation at the end of the experiment (OIV452_6dpi) shifted from 3.5 (predominant patches of dense sporulation) to 7.2 (small spots with sparse sporangiophores).

Classification of the progeny based on the symptom of sporulation was also performed under natural environmental conditions by inspecting a single leaf per plant that was artificially inoculated and remained attached to the plant for the entire course of the experiment. The progeny was scored weekly during a 3-week period after inoculation, by qualitative scoring of the symptom ( 1 present, 0 absent) on the infected leaf and by the sum of the scores per offspring, across the period of observation. The phenotypic distribution of the progeny for this parameter is reported in Fig. 2b. If the threshold for resistance is set at $\leq 1$, then $56 \%$ of the progeny rated resistant in 2004 . Some $83 \%$ of individuals that had rated $\leq 1$ in the previous year rated $\leq 1.5$ in 2005 . The overall Pearson correlation among years was 0.70 . Analysis of variance was performed using data from the two biological replicates and confirmed a significant genotypic effect for both years.

Mature plants in the vineyard allowed the classification of the progeny, exposed to natural infections, by the number of leaves with sporulating lesions within the canopy. Individuals of the progeny showed a range of variation from 0 to 32 sporulating leaves in the season of 2007 and from 0 to 24 in the season of 2008. When individuals were grouped into classes, the width of each class being four sporulating leaves, the distribution of the progeny split in 58\% of offspring included in the lowest class with 1-4 sporulating leaves, and the remainder bearing from 5 to 32 infected leaves in 2007 (Fig. 2c). The range of variation was narrower in 2008, with phenotypic differences flattened as a consequence of the increase of frequency in the intermediate classes with 5-20 infected leaves. Pearson correlation among years was 0.56 . Data from two biological replicates confirmed a significant genotypic effect in both years.

Transgressive segregation was observed for all quantitative ratings of downy mildew resistance. Extreme phenotypes outside of the range comprised between the values of the parental lines were observed in the segregating population (Fig. 2).

Dissection of genetic components controlling

$P$. viticola resistance

\section{Genetic mapping of $H R$}

Genetic maps of 'Bianca' and 'Chardonnay' were established using a pseudo test cross model. Parental maps 
included 312 and 289 SSR markers, respectively, with average marker density of one marker every $3.1 \mathrm{cM}$ and $3.3 \mathrm{cM}$. Marker order was conserved between the maps. Further details of parental maps are given in Supplementary Material S4. The chart of the 19 linkage groups of the consensus map, which merged 406 markers, is reported in Supplementary Material S3. Parental maps ensured a good coverage of all chromosomes. Four intervals larger than $20 \mathrm{cM}$ were not covered by markers on 'Bianca' linkage groups $1,6,8$, and 17, with the largest gap of $30.7 \mathrm{cM}$ between markers VVS5 and UDV085 on LG 6. Chromosome 19 split into two linkage groups. Four gaps larger than $20 \mathrm{cM}$ are present on 'Chardonnay' linkage groups 6 , 11,14 , and 18, the latter being the largest and spanning $33.7 \mathrm{cM}$ between markers VVSCU10 and CS1H010F23F. The phenotypic locus for HR mapped to the distal part of 'Bianca' LG18 in a 2.9 interval comprised between the markers UDV305 and VMC7F2. Alleles inherited from the susceptible parent did not affect HR.

UDV305, the upper border of the HR locus, is an SSR marker (sense primer TGGTGCAATGGTCATAATTT, antisense primer GAGGAAAAGAGAAAGCAAAGA) developed on the BAC clone VVCS1H060E17 end (GenBank Accession no. CT495871) of the 'Cabernet Sauvignon' physical contig 2067 (Moroldo et al. 2008). The physical contig 2067 and other contigs placed in its proximity on the physical map, such as contig 133 and contig 714, contained NBS-LRR genes (Moroldo et al. 2008). In particular, contig 714 was positive for the resistance gene analogue Vrip064, a NBS-LRR marker previously found in association with downy mildew resistant species (Di Gaspero and Cipriani 2002) and genetically mapped to the region that now spans the Rpv3 locus, along with several other RGA markers (Di Gaspero et al. 2007).

\section{QTL analysis of $P$. viticola development: mycelium growth and sporulation}

A QTL with a large phenotypic effect was identified in 'Bianca' chr18 for each parameter used for measuring quantitative characteristics of resistance to $P$. viticola (Table 2). The confidence interval for each QTL spanned the chromosomal region in which the gene for HR was localised (Fig. 3). In most cases, peaks of LOD score pointed into the HR interval delimited by the closest flanking markers.

The area covered by sporulation at 4-6 dpi scored in the leaf disc assay on leaves from greenhouse-grown plants (OIV452) was explained by a QTL with a confidence interval of $2.9 \mathrm{cM}$. Borders of the interval exactly overlapped the flanking markers closest to the HR locus, with the percentage of explained variation ranging from a maximum of $72.8 \%$ at $4 \mathrm{dpi}$ to $68.4 \%$ at $6 \mathrm{dpi}$. A QTL with the same 2.9-cM confidence interval was obtained for the integration of scores over the course of infection (OIV452_PC) and explained an even larger variance $(74.6 \%)$. Variation in sporangiophore density within the sporulating patches (SD_5dpi) was accounted for by a QTL with a confidence interval of $3.9 \mathrm{cM}$ entirely covering the HR locus and explaining $74.6 \%$ of the phenotypic variance observed at $5 \mathrm{dpi}$. The number of sporangia released per unit of inoculated area at 6 dpi was largely explained by a QTL with a confidence interval of $5.4 \mathrm{cM}$ astride the HR locus, upon inoculation with the E-F isolate. Only confidence intervals of QTLs for the extent of mycelium growth, sporangia concentration, and the OIV452 parameter upon infection with the NE-I isolate extended over a larger region. This larger interval spanned the HR interval at the Rpv3 locus and further upstream across the Rpv2 locus identified in $M$. rotundifolia by Wiedemann-Merdinoglu et al. (submitted).

All QTLs scored in the leaf disc assay from field grown plants had wider confidence intervals, and their LOD scores and percentages of explained variation were far lower (Fig. 3). This result can be partially explained by a higher variability of the physiological state of leaves, as they were detached from plants grown in uncontrolled environment, and thus subjected to a more severe effect of the environmental variance.

In addition to the QTL on chr18, a QTL just above the threshold of significance was detected on chr7 in 'Bianca' in the leaf disc assay from greenhouse-grown plants. This minor QTL explained 3.8\% of the phenotypic variance for sporangia released per unit of inoculated area and $12.1 \%$ of the phenotypic variance for sporangiophore density within sporulating patches (SD_5dpi).

A second minor QTL was identified on chr5 in the susceptible parent 'Chardonnay', which explained 24.5\% of the phenotypic variance for the extent of mesophyll invasion at 4 dpi.

For all QTLs identified by interval mapping, the closest marker to the peak was selected as a cofactor. MultipleQTL interval mapping did not detect other QTLs contributing to the observed phenotypic variance. Significance of the linkage between markers underneath the QTLs and the corresponding trait was confirmed by a Kruskal-Wallis test.

$Q T L$ analysis of quantitative scores of $P$. viticola in intact plants

The ability of the pathogen to sporulate on intact leaves artificially inoculated but still attached to the plant, grown before and after inoculation under outdoor conditions, depended on the host genotype of the offspring and was largely explained (50\% of the phenotypic variance in 2004 
Table 2 Location, significance, and confidence interval of QTLs identified by Interval Mapping in 'Bianca' and 'Chardonnay'

\begin{tabular}{|c|c|c|c|c|c|c|c|c|c|}
\hline \multirow[t]{2}{*}{ Trait } & \multicolumn{5}{|c|}{ QTL position } & \multirow[t]{2}{*}{ LOD } & \multicolumn{2}{|c|}{ LOD threshold GW } & \multirow[t]{2}{*}{$R^{2}$} \\
\hline & LG & Map & Peak (cM) & Nearest marker & $\begin{array}{l}\text { Confidence interval } \\
\text { (cM) }\end{array}$ & & $\alpha 0.2$ & $\alpha 0.05$ & \\
\hline \multicolumn{10}{|l|}{ Leaf disc assay (isolate) } \\
\hline OIV452_3dpi (E-F) & 18 & BIA & 79.5 & VMC7F2 & $75.1-86.3$ & $6.9-7.5-8.0$ & 2.1 & 2.7 & 29.2 \\
\hline OIV452_4dpi (E-F) & 18 & BIA & 77.6 & UDV305 & $76.6-79.5$ & 27.0-27.1-29.7 & 3.0 & 2.2 & 72.8 \\
\hline OIV452_5dpi (E-F) & 18 & BIA & 77.6 & UDV305 & $76.6-79.5$ & $26.3-25.2-28.1$ & 2.9 & 2.2 & 71.0 \\
\hline OIV452_6dpi (E-F) & 18 & BIA & 77.6 & UDV305 & $76.6-79.5$ & $23.5-23.5-26.1$ & 2.8 & 2.1 & 68.4 \\
\hline OIV452_PC (E-F) & 18 & BIA & 77.6 & UDV305 & $76.6-79.5$ & $28.2-28.3-31.2$ & 2.1 & 2.9 & 74.6 \\
\hline \multirow[t]{2}{*}{ SD_5dpi (E-F) } & 18 & BIA & 78.6 & VMC7F2 & $76.6-80.5$ & $28.8-29.7-30.9$ & 2.1 & 2.9 & 74.3 \\
\hline & 7 & BIA & 44.2 & UDV097 & $39.4-49.1$ & $2.0-2.0-3.1$ & 2.1 & 2.9 & 12.7 \\
\hline \multirow[t]{2}{*}{ SPORANGIA CM ${ }^{-2}$ (E-F) } & 18 & BIA & 76.6 & UDV305 & $74.1-79.5$ & $22.2-23.0-24.7$ & 2.1 & 2.8 & 65.5 \\
\hline & 7 & BIA & 44.2 & UDV097 & $34.9-50.1$ & $2.0-2.0-3.0$ & 2.1 & 2.8 & 12.1 \\
\hline OIV452_6dpi (NE-I) & 18 & BIA & 69.4 & VMCNG2F12 & $63.5-84.5$ & $1.7-1.9-3.0$ & 2.1 & 2.7 & 11.3 \\
\hline SPORANGIA CM ${ }^{-2}$ (NE-I) & 18 & BIA & 81.5 & VMC7F2 & $64.5-86.3$ & $2.7-2.7-3.9$ & 2.0 & 2.5 & 15.3 \\
\hline \multirow[t]{2}{*}{ MG_4dpi (NE-I) } & 18 & BIA & 79.5 & VMC7F2 & $67.2-86.3$ & $3.1-3.1-4.3$ & 2.2 & 2.8 & 16.2 \\
\hline & 5 & CHA & 24.3 & CS1E104J11F & $10.6-39.2$ & $2.0-2.1-3.1$ & 2.2 & 3.0 & 12.1 \\
\hline \multicolumn{10}{|l|}{ Inoculation on intact leaf } \\
\hline \multirow[t]{2}{*}{ ¿ SPORULATION_2004 } & 18 & BIA & 78.6 & VMC7F2 & $75.1-81.5$ & $15.6-15.7-16.7$ & 2.1 & 2.9 & 50.0 \\
\hline & 7 & BIA & 44.2 & UDV097 & $33.9-47.1$ & $2.0-2.2-3.2$ & 2.1 & 2.9 & 12.3 \\
\hline$\Sigma$ SPORULATION_2005 & 18 & BIA & 79.5 & VMC7F2 & $76.6-82.5$ & $32.7-39.6-40.6$ & 2.1 & 2.8 & 80.5 \\
\hline \multicolumn{10}{|l|}{ Natural infection on whole plant } \\
\hline No.S.LEAVES_2007 & 18 & BIA & 79.5 & VMC7F2 & $73.1-82.5$ & $15.6-15.8-17.2$ & 2.0 & 2.6 & 50.0 \\
\hline No.S.LEAVES_2008 & 18 & BIA & 80.5 & VMC7F2 & $72.1-85.5$ & $8.1-8.5-10.0$ & 2.7 & 2.2 & 33.9 \\
\hline
\end{tabular}

The OIV452 index reflects severity of symptoms scored daily and cumulatively over the course of infection (OIV452_PC). SPORANGIA CM ${ }^{-2}$ is a count of sporangia released at 6 dpi per unit of inoculated leaf area. SD_5dpi is a categorical rating of sporangiophore density at 5 dpi, and mycelium growth at $4 \mathrm{dpi}$ is a categorical rating based on the extent of mesophyll invasion by the mycelium at 4 dpi (MG_4dpi). $\Sigma$ SPORULATION reports the sum of weekly scores of presence/absence of sporulation, recorded during a 3-week period after inoculation in an artificially infected leaf, attached to the plant grown and incubated outdoors, in 2 years of observations (2004 and 2005). The number of sporulating leaves (No.S.LEAVES) is based on counts of leaves with fungal sporulation in unsprayed vines in the open field, in 2 years of observations (2007 and 2008)

$E-F$ indicate artificial inoculation with a $P$. viticola isolate collected in Eastern France, $N E-I$ indicate artificial inoculation with an isolate collected in North-Eastern Italy

and $80.5 \%$ in 2005) by a QTL spanning a confidence interval of 4.5 and $5.9 \mathrm{cM}$ across the HR locus. A minor QTL that explained a further $12.3 \%$ of phenotypic variance was identified on Bianca chr7 in the season 2004. This QTL spanned the same region identified in the leaf disc assay for sporangiophore density and number of sporangia per unit of leaf area.

\section{QTL analysis of $P$. viticola infection in open field}

Phenotypic analysis carried out in vines grown in an open field confirmed the QTL in the chromosome 18 of 'Bianca'. The QTL peaked at 79.5 and $80.5 \mathrm{cM}$ in 2007 and 2008, with percentage of explained variance of 50.0 and $33.9 \%$, respectively. The LOD peak was less sharp and the confidence interval wider than those obtained in analyses of plants grown in a controlled environment.

\section{Discussion}

Phenotypic segregation of downy mildew resistance in 'Bianca' resulted in a single dominant allele at the Rpv 3 locus on chr18 that explained most of the phenotypic variance. $R p v 3$ was confined in the interval between markers UDV305 and VMC7F2, which are $2.9 \mathrm{cM}$ apart. The resistant allele at Rpv3 confers the ability to mount an HR within $48 \mathrm{~h}$ post artificial inoculation in detached leaves, corresponding to the pathogen's stages of colonisation of the substomatal cavity and establishment of biotrophy in the mesophyll. In addition to the presence of necrotic spots as a result of the host reaction, the other phenotypic effects of the Rpv 3 allele consisted in a quantitative reduction of pathogen performance. The progress of pathogen development into the next stages of its cycle depended on the environmental conditions in which the host-pathogen interaction took place. 


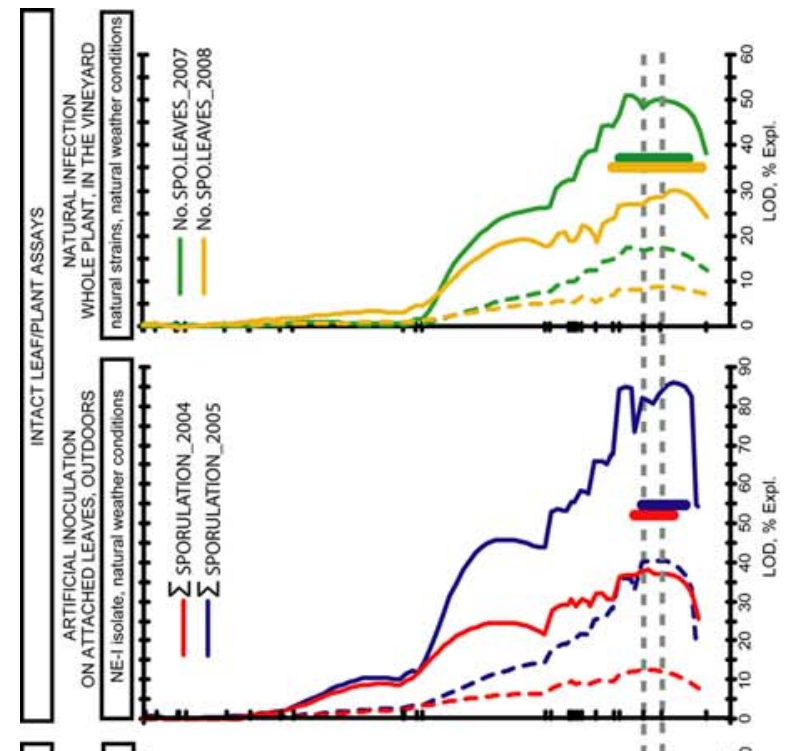

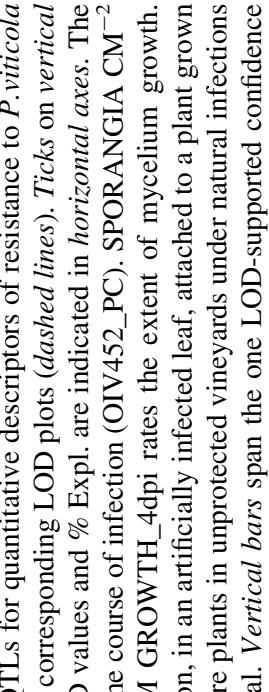

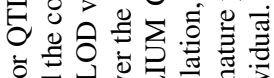

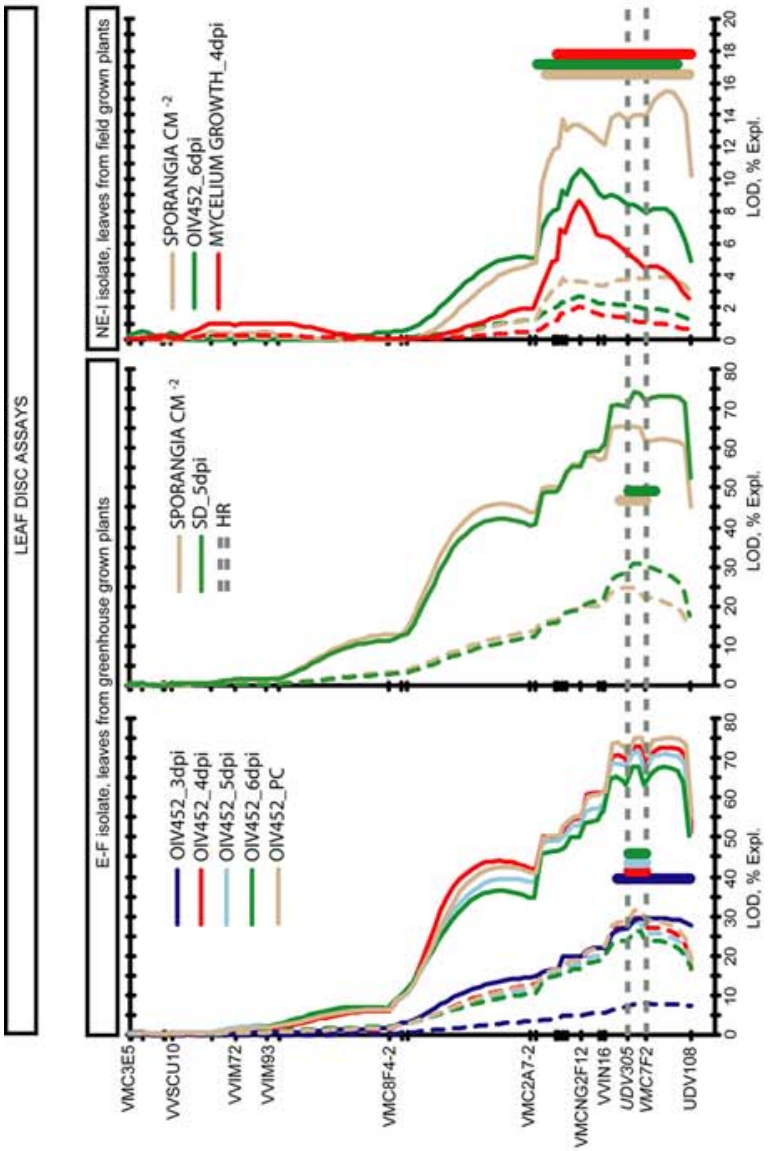

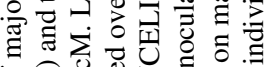

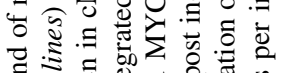

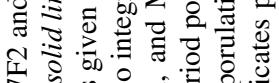

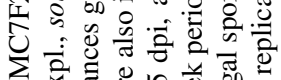

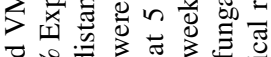

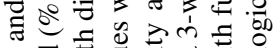

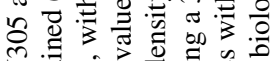

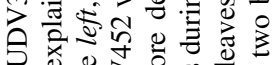

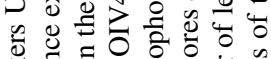

㐘

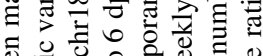

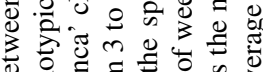

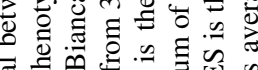

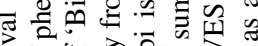

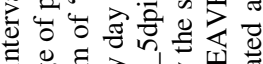

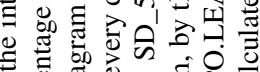

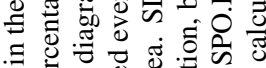

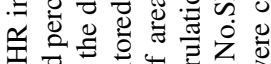

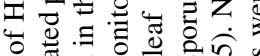

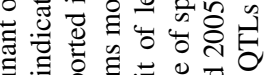

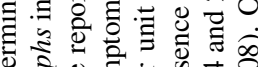

क्षे

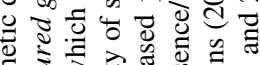

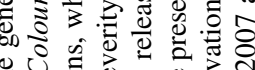

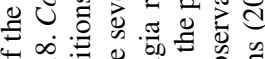

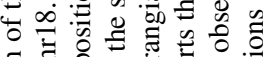

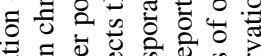

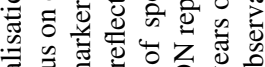

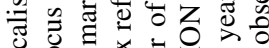

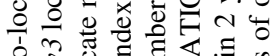

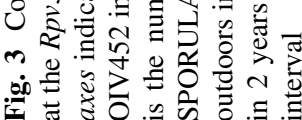


Under the most favourable conditions for the pathogen, which is on shoots with tender leaves such as those detached from plants grown in a controlled environment, with a high concentration of artificial inoculum, optimum temperature, and relative humidity prior to and during incubation, some hyphae were able to emerge from the necrotised mesophyll tissue, to colonise at a limited extent in the surrounding intercellular space, and to produce sparse sporulation. However, a significant decrease in disease severity was witnessed by a reduction of mesophyll area invaded by the mycelium at $4 \mathrm{dpi}$, a reduction of the sporangiophore density at $5 \mathrm{dpi}$, and a reduction of the number of sporangia released per unit of leaf area at the end of infection at 6 dpi. A large part of the phenotypic variance observed for these parameters (up to $74.6 \%$ ) was explained by coincident QTLs, with confidence intervals always spanning the Rpv3 locus.

Under natural conditions, where the host plants are grown in a harsher environment and exposed to naturally occurring concentrations of $P$. viticola, and the incubation occurs under fluctuating weather conditions that might alternatively favour either partner, the plant reaction triggered by the Rpv3 allele translated into a more severe limitation of pathogen spread. Under these circumstances, absence of sporulation or reduced numbers of leaves with sporulating lesions were observed in resistant individuals in the open field, and a QTL spanning the Rpv3 locus explained from 33.9 to $50 \%$ of the phenotypic variance over 2 years.

The variability of the experimental material, possibly due to the physiological status of the host leaf, concentration of the inoculum, and environmental conditions prior to and during the course of the infection, must be minimised when the identification of genetic determinants of resistance is the goal of the study, because the experimental error in phenotypic ratings of resistance could alter the precise positioning of the genetic determinant/s. Standardised and rigorous experimental protocols, and the choice of measurable parameters and appropriate classes for categorical scores are all necessary for reducing the experimental error.

The leaf disc assay applied to leaves detached from cuttings grown in greenhouses (Brown et al. 1999; DíezNavajas et al. 2008) revealed three advantages in this study. First, it allowed the detection of the occurrence of localised necroses at the infection sites in resistant genotypes, and the mapping of the locus responsible for the HR. This phenotype was less detectable on field plant foliage as it could be sometimes confused with necrotic spots caused by other biotic or abiotic stresses. Second, it reduced a considerable part of the environmental variance, thus narrowing the confidence interval of QTLs in quantitative scores of pathogen growth. For instance, the one LOD-supported QTL interval for severity of disease symptoms, as recorded by the OIV452 descriptor, spanned $2.9 \mathrm{cM}$, with borders of the confidence interval exactly overlapping the flanking markers of the HR locus. In field scores, ratings based on the OIV452 descriptor identified the QTL in the same region, but did not allow the restriction of the confidence interval into less than 9.4$13.4 \mathrm{cM}$.

Leaf disc assay had the further benefit of allowing ratings of host reaction and pathogen development to be taken daily, during a controlled progression of the infection. The dynamic monitoring of the evolution of infection allowed the dissection of the sequence of events contributing to the final resistant phenotype, based on their time of occurrence. Phenotypic dissection of the trait into its components resulted in a mendelization of the quantitative resistance observed in the progeny.

In this way, we were able to demonstrate that the earliest phenotypic effect caused by the Rpv3 locus is an HR reaction in the resistant individuals. All subsequent stages of pathogen development are adversely affected almost exclusively by the Rpv3 resistant allele. Similar type and timing of the host reaction were also observed in the grapevine 'Solaris' (Gindro et al. 2003). A minor QTL on chr7 was consistently scored for the extent of pathogen growth and sporulation, which explained a limited part of the residual phenotypic variance. This architecture of the genetic control is in agreement with early investigations into downy mildew resistance in North American grapes, which predicted a prominent monofactorial component responsible for the hypersensitive response, and additional quantitative components exerting some influence on pathogen growth at later phases of the interaction (Boubals 1959).

For breeding purposes, it is essential to know how genetic factors identified in controlled experiments translate into field resistance. Parallel evaluation of the same progeny in leaf disc assays and on intact plants under natural conditions confirmed that the 'Bianca' Rpv3 resistant allele significantly reduced the number of sporulating leaves, without eradicating the pathogen but allowing the plant to survive the infection. A wider spectrum of variation in resistance levels was observed in the field between biological replicates and between genotypes. This could be the result of a number of factors, such as uneven dispersion of inoculum, heterogeneous composition of the natural inoculum mixtures across the experimental plot, and genome-wide heterozygosity of individuals for many physiological characteristics, which could affect the magnitude of disease symptoms in each class of genotypes at the Rpv3 locus, in addition to the genotype $\times$ environment interaction of each offspring under uncontrolled conditions before and during the infection. 
Grapevine accessions are commonly rated for downy mildew resistance by scoring disease symptoms on young leaves (Cadle-Davidson 2008). Under natural conditions, distal leaves that continuously emerge and expand beneath the apex are the organs where overwintering oospores give rise to reiterate oosporic infections, through asynchronous cycles of germination during shoot elongation (Kennelly et al. 2005a). Leaf tissues of susceptible hosts might also be home to cycles of vegetative propagation, with a limited number of genotypes arisen from oosporic infections that substantially contribute to the sporangial epidemics of the disease (Gobbin et al. 2005). The disease may injure other organs at specific developmental stages, e.g., by curling cluster stems before blooming, damaging flower buttons at blooming, and causing infected tissues to eventually wither. After fruit set, sporangia may be splashed from nearby leaf lesions onto developing berries. Fruit exposure to $P$. viticola attacks is temporally limited to the developmental stages when functional stomata on berry epidermis and the pedicels open the way to the inner tissues (Kennelly et al. 2005b). This condition lasts in pea-sized fruit until stomata are converted to lenticels. Fruit damage is correlated with the level of leaf susceptibility, the leaf lesions being a permanent source of sporangial inoculum, in presence of favourable weather conditions. Welter et al. (2007) detected overlapping QTLs for leaf resistance and berry resistance on chr18 in 'Regent'. Nevertheless, some exceptions to this general observation are reported in literature. 'Chancellor' has susceptible fruit in presence of healthy foliage, while 'Delaware' has susceptible foliage but healthy bunches (reviewed in Kennelly et al. 2005a, b). In our field experiment, 'Bianca' descendents displayed a consistent association between leaf resistance and health of the clusters across the genotypes (data not shown).

The Rpv3 locus resides in the distal part of chr18, which is very rich in TIR-NBS-LRR genes (Di Gaspero et al. 2007). The marker UDV305 that flanks the HR locus on the proximal side was placed in a physical contig that contains NBS-LRR genes (Moroldo et al. 2008). The evidence that resistance is based on HR and that the class of genes clustered at the Rpv3 locus are NBS-LRRs leads to the expectation that downy mildew resistance inherited by 'Bianca' from North American grape is race-specific. In this study, resistance ratings of the progeny were consistent between independent infections with two isolates, one collected in France and the other in Italy. However, such a gene-for-gene host resistance might be defective against virulent strains that escape recognition, as found in 'Regent' (Kast et al. 2001), which shares with 'Bianca' the resistant ancestor 'Villard blanc'. Screening of wild North American grapevines and genotypes selected from their deliberate crosses, involving at least one resistant parent, identified $16 \%$ of resistant accessions to a single isolate being susceptible to a second isolate (Cadle-Davidson 2008), when just two pathogen strains were tested. This provides further clues to the strain specific nature of downy mildew resistance in North American species.

Genetic mapping of the Rpv3 locus based on the qualitative HR trait allowed us to distinguish it from the Rpv2 locus mapped in $M$. rotundifolia (Wiedemann-Merdinoglu et al. submitted) in a proximal region on the same chromosome. BlastN projection of the marker sequences bordering the 'Bianca' Rpv3 genetic interval and the M. rotundifolia Rpv2 interval on the PN40024 grape sequence (Jaillon et al. 2007) identifies two regions that are separated by approximately $1.5 \mathrm{Mbp}$ on chr18.

Acknowledgments This research was supported by funds from the Italian Ministry of Agriculture, VIGNA project; from the Italian Ministry of Education and Research PRIN2006 no. 2006073137; from the Regional Government of Friuli Venezia Giulia, Grape Breeding Project; from INRA and the ANR GrapeSeq project. We thank P. Coste, G. Comuzzo, and R. Frezza for technical assistance in plant and inoculum maintenance, D. Copetti for help in field scorings, and C. Coleman for proofreading the manuscript.

\section{References}

Anonymous (1983) Descriptor list for grapevine varieties and Vitis species. Office International de la Vigne et du Vin (OIV), Paris

Barker CL, Donald T, Pauquet J, Ratnaparkhe A, Bouquet A, AdamBlondon A-F, Thomas MR, Dry I (2005) Genetic and physical mapping of the grapevine powdery mildew resistance gene, Run1, using a bacterial artificial chromosome library. Theor Appl Genet 111:370-377

Boubals D (1959) Amélioration de la résistance de la vigne au mildiou (Plasmopara viticola (Berk et Curt.) Berlese et de Toni). Recherche de géniteurs de résistance. Annales de l'Amélioration des Plantes 6:481-525

Brown MV, Moore JN, Fenn P, McNew RW (1999) Comparison of leaf disk, greenhouse and field screening procedures for evaluation of grape seedlings for downy mildew resistance. Hortscience 34:331-333

Cadle-Davidson L (2008) Variation within and between Vitis spp. for foliar resistance to the downy mildew pathogen Plasmopara viticola. Plant Dis 92:1577-1584

Cipriani G, Di Gaspero G, Canaguier A, Jusseaumes J, Tassin J, Lemainque A, Roux C, Adam-Blondon A-F, Testolin R (2009) Molecular linkage maps: strategies, resources and achievements. In: Zapater MM, Adam-Blondon AF (eds) Grapes. In: Chittaranjan Kole (Series ed) Series on "Genomics of fruit and vegetables crops". Sciences Publishers, Enfield NH, USA

Csizmazia J, Bereznai L (1968) A szõlõ Plasmopara viticola és a Viteus vitifolii elleni rezisztencia nemesités eredményei. Orsz Szõl Bor Kut Int Évkönyve, Budapest:191-200

Dai GH, Andary C, Mondolot-Cosson L, Boubals D (1995) Histochemical studies on the interaction between three species of grapevine, Vitis vinifera, V. rupestris and V. rotundifolia and the downy mildew fungus, Plasmopara viticola. Physiol Mol Plant Pathol 46:177-188

Di Gaspero G, Cipriani G (2002) Resistance gene analogs are candidate markers for disease-resistance genes in grape (Vitis spp.). Theor Appl Genet 106:163-172 
Di Gaspero G, Cipriani G, Adam-Blondon A-F, Testolin R (2007) Linkage maps of grapevine displaying the chromosomal locations of 420 microsatellite markers and 82 markers for $R$-gene candidates. Theor Appl Genet 114:1249-1263

Díez-Navajas AM, Greif C, Poutaraud A, Merdinoglu D (2007) Two simplified fluorescent staining techniques to observe infection structures of the oomycete Plasmopara viticola in grapevine leaf tissues. Micron 38:680-683

Díez-Navajas AM, Wiedemann-Merdinoglu S, Greif C, Merdinoglu D (2008) Nonhost versus host resistance to the grapevine downy mildew, Plasmopara viticola, studied at the tissue level. Phytopathology 98:776-780

Doligez A, Adam-Blondon AF, Cipriani G, Di Gaspero G, Laucou V, Merdinoglu D, Meredith CP, Riaz S, Roux C, This P (2006) An integrated SSR map of grapevine based on five mapping populations. Theor Appl Genet 113:369-382

Eibach R, Töpfer R (2003) Success in resistance breeding: 'Regent' and its steps into the market. Acta Hort 687-691

Fischer BM, Salakhutdinov I, Akkurt M, Eibach R, Edwards KJ, Töpfer R, Zyprian EM (2004) Quantitative trait locus analysis of fungal disease resistance factors on a molecular map of grapevine. Theor Appl Genet 108:501-515

Gindro K, Pezet R, Viret O (2003) Histological study of the responses of two Vitis vinifera cultivars (resistant and susceptible) to Plasmopara viticola infections. Plant Physiol Biochem 41:846853

Gobbin D, Jermini M, Loskill B, Pertot I, Raynal M, Gessler C (2005) Importance of secondary inoculum of Plasmopara viticola to epidemics of grapevine downy mildew. Plant Pathol 54:522-534

Iwanov J, Waltschev W (1973) The inheritance of features of cold hardiness and Plasmopara resistance by interspecific hybridization of vines. International Symposium of vine breeding. Geilweilerhof, Siebeldingen, Germany, 25-29 September 1973

Jaillon O, Aury J-M, Noel B, Policriti A, Clepet C et al (2007) The grapevine genome sequence suggests ancestral hexaploidization in major angiosperm phyla. Nature 449:463-468

Jürges G, Kassemeyer H-H, Dürrenberger M, Düggelin M, Nick P (2009) The mode of interaction between Vitis and Plasmopara viticola Berk. \& Curt. Ex de Bary depends on the host species. Plant Biol. doi:10.1111/j.1438-8677.2008.00182.x

Kast WK, Stark-Urnau M, Seidel M, Gemmrich AR (2001) Interisolate variation of virulence of Plasmopara viticola on resistant vine varieties. Bull OILB/SROP 24:45-49

Kennelly MM, Eugster C, Gadoury DM, Smart CD, Seem RC, Gobbin D, Gessler C (2005a) Contributions of oospore inoculum to epidemics of grapevine downy mildew (Plasmopara viticola). Phytopathology 94:S50

Kennelly MM, Gadoury DM, Wilcox WF, Magarey PA, Seem RC (2005b) Seasonal development of ontogenic resistance to downy mildew in grape berries and rachises. Phytopathology 95:14451452

Kortekamp A, Zyprian E (2003) Characterization of Plasmopararesistance in grapevine using in vitro plants. J Plant Physiol 160:1393-1400

Marguerit E, Boury C, Manicki A, Donnart M, Butterlin G, Némorin A, Wiedemann-Merdinoglu S, Merdinoglu D, Ollat N, Decroocq S (2009) Genetic dissection of sex determinism, inflorescence morphology and downy mildew resistance in grapevine. Theor Appl Genet 118:1261-1278

Merdinoglu D, Wiedemann-Merdinoglu S, Coste P, Dumas V, Haetty A, Butterlin G, Greif C (2003) Genetic analysis of downy mildew resistance derived from Muscadinia rotundifolia. Acta Hort 603:451-456

Moroldo M, Paillard S, Marconi R, Fabrice L, Canaguier A, Cruaud C, De Berardinis V, Guichard C, Brunaud V, Le Clainche I, Scalabrin S, Testolin R, Di Gaspero G, Morgante M, AdamBlondon AF (2008) A physical map of the heterozygous grapevine 'Cabernet Sauvignon' allows mapping candidate genes for disease resistance. BMC Plant Biol 8:66

Staudt G, Kassemeyer HH (1995) Evaluation of downy mildew resistance in various accessions of wild Vitis species. Vitis $34: 225-228$

Unger S, Büche C, Boso S, Kassemeyer HH (2007) The course of colonization of two different Vitis genotypes by Plasmopara viticola indicates compatible and incompatible host-pathogen interaction. Phytopathology 97:780-786

Van Ooijen JW (2004) MapQTL 5, Software for the mapping of quantitative trait loci in experimental populations. Kyazma B.V., Netherlands

Welter LJ, Göktürk-Baydar N, Akkurt M, Maul E, Eibach R, Töpfer R, Zyprian EM (2007) Genetic mapping and localization of quantitative trait loci affecting fungal disease resistance and leaf morphology in grapevine (Vitis vinifera L). Mol Breed 20:359374

Zyprian E, Akkurt M, Fischer B, Salakhutdinov, Welter L, Kortekamp A, Eibach R, Töpfer R (2005) Fundamental research meets practical breeding: genetics of disease resistance in grapevine. In: W Qiu and LG Kovacs (eds) Proceedings of the International Grape Genomics Symposium, July 12-14, 2005. St. Louis, Missouri, pp 163-168 